\title{
Mitteilungen/Communications
}

\section{Forschungsbeiträge der Schweizerischen} Stiftung für Kardiologie.

Die Schweizerische Stiftung für Kardiologie gewährt finanzielle Unterstützung für Forschungsprojekte auf dem Gebiet der Herz- und Gefässkrankheiten. Gesuche um Forschungsbeiträge sind bis 30. April 1988 (Poststempel) in fünf Exemplaren an die nachstehende Adresse zu richten, wo auch die Gesuchsformulare bezogen werden können: Schweizerische Stiftung für Kardiologie. Postfach 176, 3000 Bern 15.

\section{Contributions pour la recherche} de la Fondation suisse de cardiologie La Fondation suisse de cardiologie assure un soutien financier à des projets de recherche dans le domaine des maladies cardio-vasculaires. Les demandes de subsides doivent être adressées, en cinq exemplaires, jusqu'au 30 avril 1988 (date du cachet postal) à l'adresse ci-dessous, où l'on peut aussi se procurer les formulaires nécessaires. Fondation suisse de cardiologie, case postale 176. 3000 Berne 15.

\section{Tagungen/ Congrès}

\begin{abstract}
Université de Nancy l, faculté de médecine, département de santé publique, Formation en santé publique et santé communautaire Formation continue, enseignement universitaire de 3ème cycle 1987-1988. création 1988: Enseignement à distance. Renseignements: Secrétariat du Professeur J.P. Deschamps, Faculté de Médecine, Départment de Santé Publique, B.P. 184, F-54505 Vandœuvre-lès-Nancy Cédex, Tél.: 83565656 83514415 poste 1350
\end{abstract}

\footnotetext{
Der psychosomatische Zugang - Chance für Patient und Arzt

Ascona Gespräche, Monte Verità, 25.-27. März 1988. Wissenschaftliche Leitung und Auskünfte: Prof. Dr. med. B. Lu-
}

Pressemitteilung der Schweizerischen Psoriasis-Gesellschaft

Die Schweizerische Psoriasis-Gesellschaft (SPG), im März 1974 gegründet, konnte dieser Tage ein eigenes Büro eröffnen.

Aufgabe der SPGF ist es, allen an Psoriasis (Schuppenflechte) oder Vitiligo (Weissfleckenkrankheit) erkrankten Personen zu helfen. Psoriasis ist eine nicht ansteckende, chronische Krankheit, deren Ursache in einer bis zu $15 \mathrm{mal}$ höheren Zellteilung gegenüber gesunden Menschen liegt, was zu extremer Schuppenbildung der Haut führen kann. Die betroffenen Personen sind dadurch vielen Unannehmlichkeiten ausgesetzt, wobei die Krankheit bis zur Arbeits. unfähigkeit oder gar Invalidität führen kann. Vitiligo entsteht durch Pigmentschwund auf der Haut und wird meistens als rein kosmetisches Problem abgetan. Viele Vitiligo-Patienten sind insbesondere bei Sonnenschein einer erhöhten Verbrennungsgefahr ausgesetzt und sind gezwungen, während der gesunde Mensch sich leichter bekleiden kann, gegen das Sonnenlicht schützende Kleider anzuziehen. Ebenso wird das Wandern, Sonnenbaden und der
Freiluftsport diesen Menschen im allgemeinen verunmöglicht.

Beide Patientengruppen sind durch ihr Aussehen, teilweise durch Behinderungen am Arbeitsplatz oder aber auch im Privatleben, benachteiligt und werden vielfach in die Isolation getrieben, was auch die nicht geringe Selbstmordrate unter diesen Patienten erklären könnte.

Zweck der SPG ist es. sich für die Belange aller Betroffenen einzusetzen. So will die SPG die Interessen aller Psoriasis- und Vitiligo-Patienten wahren und setzt sich für eine Zusammenarbeit im medizinischen und versicherungstechnischen Bereich ein. In den Regionalgruppen können sich die Mitglieder und Interessenten kennenlernen und offen über ihre Probleme sprechen; dies bildet zugleich die Grundlage der gesamtschweizerischen Aktivität der SPG. Die Mitglieder erhalten sechsmal jährlich eine Zeitschrift sowie interne Mitteilungen. Die SPG lässt sich dabei von anerkannten Grundsätzen der medizinischen Wissenschaft leiten.

Unterlagen sind bei: SPG, Postfach 507, 8027 Zürich, erhältlich.
ban-Plozza, Piazza Pedrazzini, 6600 Locarno

Symposium «Ernährung und Gesundheit» 1988

13.-15. April 1988, Davoser Kongresszentrum. Stiftung Forum Davos, Postfach, 7270 Davos Platz, Tel, 083/3 7102

The International Congress for Infectious Diseases

April 17th-21st 1988. ISID, 180 Longwood Avenue, Boston, MA 02115. USA. Tel. (617) $732-2270$

\section{Congrès de Pneumologie de Langue \\ Française}

9-11 juin 1988. Secrétariat du Pr. Paul Léophonte. Hôpital Purpan, F-31059 Toulouse Cédex

Fifth World Conference on Lung Cancer Interlaken, Switzerland, August 28th-September 1st, 1988. Scientific Secretariat: Rudolf A. Joss MD. Institute for Medical Oncology, University of Bern, Inselspital, 3010 Bern, Tel. 031/64 2438

\section{Jahreskongress 1988}

2.-4. September 1988, Jahreskongress der Schweizerischen Gesellschaft für Allgemeinmedizin SGAM/SSMG, Kongresshaus Zürich. Themenschwerpunkt: Fortbildung in der Geriatrie. Wissenschaftliches Sekretariat: Dr. med. H. Reimann. Im Haufland 18, 8627 Grüningen. Administratives Sekretariat: E. Haase, Helvetierstrasse 24. 4106 Therwil, Tel. 061/733236

XIIth International Congress for Tropical Medicine and Malaria

September 18th-23rd, 1988, International Congress Centre RAI Amsterdam, The Netherlands. Mailing address: c/o RAI Organisatie Bureau Amsterdam bv, Europaplein 12. NL-1078 GZ Amsterdam, The Netherlands. Tel. (31)20-5491212

ERGO-IA'88 - Ergonomie et Intelligence Artificielle

4-6 octobre 1988, Biarritz, France. Secrétariat du colloque: ERGO-IA'88, 156, boulevard Péreire, F-75017 Paris, Tél. (1) 47 662419

\section{Voranzeigen/ Dates à retenir}

'The Old in a New World' The International Assuciation of Gerontology

The 14th International Congress of Gerontology will be held at The Convention Center in Acapulco. Mexico, Monday, June 19th through Friday, June 23rd 1989. Call for papers: Congress Secretariat, Jojutla No. 91. Tlalpan C.P. 14090, Mexico, D.F. / Mexico

\section{Elmia Ergonomics 89}

International Trade Fair \& Conference on Safety and Health at Work, Jönköping, Sweden. October 3rd-6th, 1989. Elmia Ergonomics 89 , Box 6066, S-550 06 Jönköping/ Sweden. Tel.: 4636119060 